\title{
Cosmic rays observations with a wide-angle cherenkov telescope
}

\section{Lev Timofeev*Yu.G.Shafer Institute of Cosmophysical Research and Aeronomy SB RAS \\ E-mail: timofeevlev@ikfia.ysn.ru}

\section{Anatoly Ivanov Yu.G.Shafer Institute of Cosmophysical Research and Aeronomy SB RAS \\ E-mail: aivanoveikfia.ysn.ru}

\begin{abstract}
A proposed new method for measuring the cherenkov light from extensive air showers (EAS) of cosmic rays (CR), which allows to determine not only the primary particle energy and angle of arrival, but also the parameters of the shower in the atmosphere - the maximum depth and "age". For measurements it is proposed to use cherenkov light produced by EAS in a ground network of wide-angle telescopes which are separated from each other by a distance 100-300 m depending on the total number of telescopes operating in coincidence, acting autonomously, or includes a detector of the charged components, radio waves, etc. as part of the EAS. Results of such an array should be developed. The energy measurement and CR angle of the arrival data on the depth of the maximum and the associated mass of the primary particle generating by EAS. This is particularly important in the study of galactic cosmic ray for $E>10^{14} \mathrm{eV}$, where currently there are no direct measurements of the maximum depth of the EAS.
\end{abstract}

35th International Cosmic Ray Conference - ICRC2017

12-20 July, 2017

Bexco, Busan, Korea

${ }^{*}$ Speaker. 


\section{Introduction}

Investigation of Cherenkov light induced by cosmic rays (CRs) cascading in the atmosphere began in the middle of the last century in the UK and the USSR. An exhaustive description of the early developments of the study of Cherenkov light is provided in [1], while recent reviews of the entire area are presented, e.g., in [2,3]. It is now well-known that the angular and temporal structure of the Cherenkov light emitted by an extensive air shower (EAS) can be used to infer the longitudinal development parameters of the shower; specifically, the lateral distribution of the light intensity measured is often used to estimate the energy and mass of the primary particle initiating EAS [4 - 7]. The angular distribution of Cherenkov photons from EAS was calculated by V.I. Zatsepin [4] assuming that it is determined primarily by that of electrons in the shower. Subsequently, Fomin and Khristansen proposed [5] to use the pulse shape of the Cherenkov signal, namely the pulse width, to indicate the showerEşs maximum position, $x_{m}$, in the atmosphere. Experimental measurements of the Cherenkov signal pulse shape were performed initially in Yakutsk and in Haverah Park [6,7]. The results were used to estimate $\mathrm{xm}$, and attempts were made to evaluate the cascade parameters of electrons at $\mathrm{CR}$ energies of approximately $10^{17} \mathrm{eV}[7,8]$. A variety of detectors were used then; for example, the Tunka experiment operates an array of Cherenkov detectors near Lake Baikal [10]. Our intention to develop a Cherenkov telescope functioning as a differential detector of EAS was motivated by the possibility to measure the depth of the cascade maximum and/or the shower age via the angular and temporal distributions of the Cherenkov signal [13]. Combining xm and the shower age with other characteristics measured with surface detectors of the EAS array, e.g. the energy and muon content, one is able to estimate the average mass composition of CRs. Experimental arguments in elucidating the origin of the knee and ankle in the CR spectrum will be significantly strengthened by the measurements of the angular and temporal distributions of the Cherenkov signal in the energy range above $10^{15} \mathrm{eV}$. Existing scenarios of CR acceleration in the sources differ in the expected model composition around the knee and in the transition region between galactic and extragalactic components [14], so accurate estimation of the average mass of CR nuclei, in addition to the improved measurement of the sharpness of the knee and ankle, should allow us to discriminate some scenarios. The latest updates with the method of registration of radio emission of ultrahigh energy particles at Yakutsk array described in [20].

\section{Data acquisition system}

The signals from each channel of the multi-anode PMTs continuously input to preamplifiers and onwards to the Analogue to Digital converters and stored in the buffer memory of a 32-channel industrial computer see Fig 1. On the arrival of a trigger generated by the EAS array, an industrial computer saves the accumulated pre-history as a separate file labeled with the time mark and the assignment of a serial number.

We carried out the photomultiplier sensitivity check from plastic scintillator in the voltage range from 900 to 1300 volts and obtained a coordinate-dependent sensitivity of the Photo Multiplier Tube from which a radial nonuniformity of sensitivity is seen Fig 2. We also measured the angular dependence of the telescope, the energy spectrum of telescope channels, and found that the Photo Multiplier Tube dark current is minimal at a voltage less than 1300 volts. Currently, the 


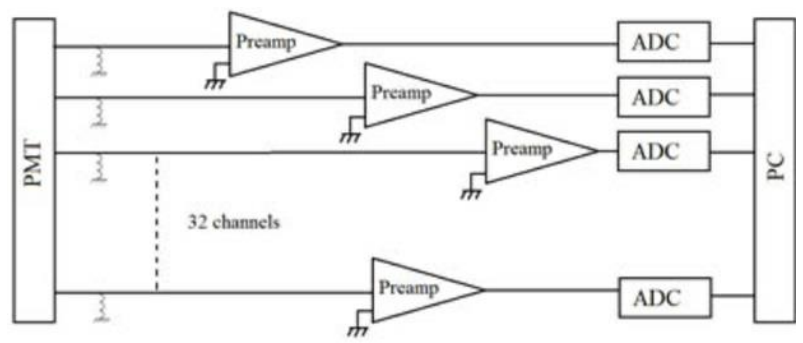

Figure 1: Data acquisition system scheme

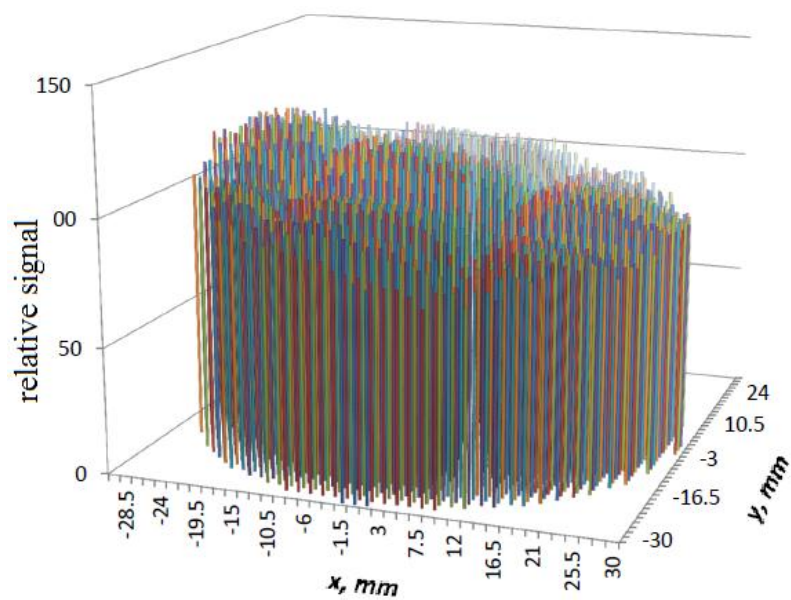

Figure 2: Normalized coordinate-dependent sensitivity of the Photo Multiplier Tube

telescope is installed at the Yakutsk EAS array at station obscura-3 at a distance 500 meters from the center of the array, where the field test is run.

An example of the output signals from the multi anode PMT is shown in Fig. 3. The average number of channels triggered in the event, where the signal is definitely exceeds the noise level is $17 \pm 6$. So we get a good accuracy of the pulse duration by means of several independent measurement channels.

\section{Pulse duration as a function of the distance to the shower core}

Here we focus on the pulse shape parameters of the Cherenkov signal from EAS. The main feature of the Cherenkov signal is its rise time with the shower core distance due to geometrical reasons. The data was accumulated during the observation period from 2012 to 2015, when 2000 coincident EAS events were detected. A sample of showers is selected in the energy interval from $10^{16}$ to $10^{19} \mathrm{eV}$. Additional cuts are applied to exclude showers with axes out of the array area and zenith angles more than sixty degrees. A specific cut is used for saturated signals where EAS events generate more Cherenkov light than the Cherenkov telescope can properly detect. These signals are also rejected. The number of EAS events surviving after cuts is 733 with the primary energy distribution shown in Fig. 4. 

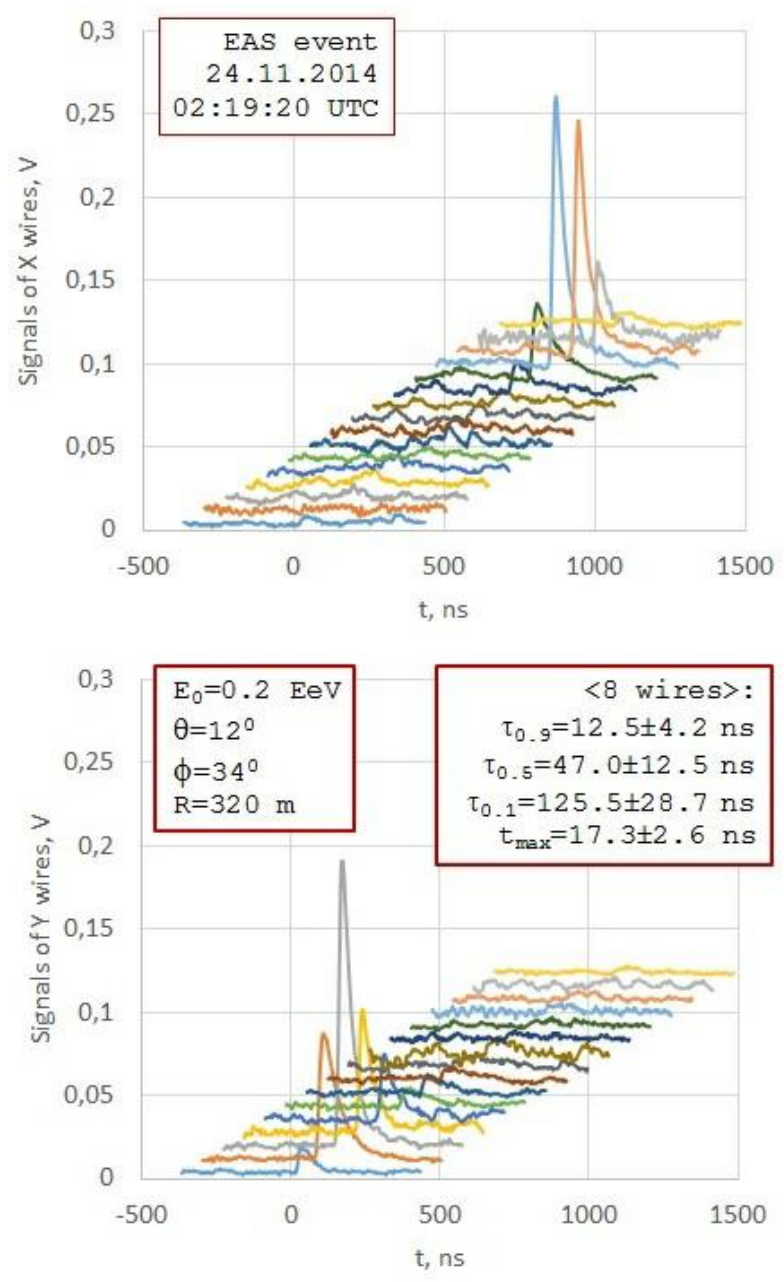

Figure 3: Signals of 32 wires from the multi-anode PMT at the focus of the telescope. The EAS parameters in a particular shower are estimated using data of the surface detectors. Pulse durations $(\tau)$ are measured at $0.1,0.5,0.9$ levels of the pulse maximum $V_{\max }$. The time difference $\left(t_{\max }\right)$ between $V_{\max }$ and $0.1 V_{\max }$ of the rising signal is shown in the right panel.

The main feature of the signal is its rise time variation with the shower core distance due to geometrical reasons. The first measurements of the parameter were made in Yakutsk [12] and Haverah Park [13]. The most recent results are provided by the Tunka array [14]. Our measurement of the full width at half-maximum of the signal confirms the previous results (see Fig.5). Event numbers are indicated above our data points. The energy ranges relevant are: Haverah Park $(\mathrm{E}) \sim$ $0.2 \mathrm{EeV}$; Yakutsk, $1975 \mathrm{E} \in(0.001,1) \mathrm{EeV}$; Tunka $\mathrm{E} \in(0.003,0.03) \mathrm{EeV}$.

We analyzed the full width at half-maximum (FWHM) dependence on other shower parameters such as zenith and azimuth angles, and energy. No significant variation versus angles is found exceeding the instrumental errors. On the other hand, an indication of the energy dependence of the pulse width is found in our data. Application of the FWHM(R) function is possible in the shower core location procedure in the array plane. The method was pioneered by John Linsley [15] using charged particle signal width vs R. While in the Yakutsk array group the particle and Cherenkov 


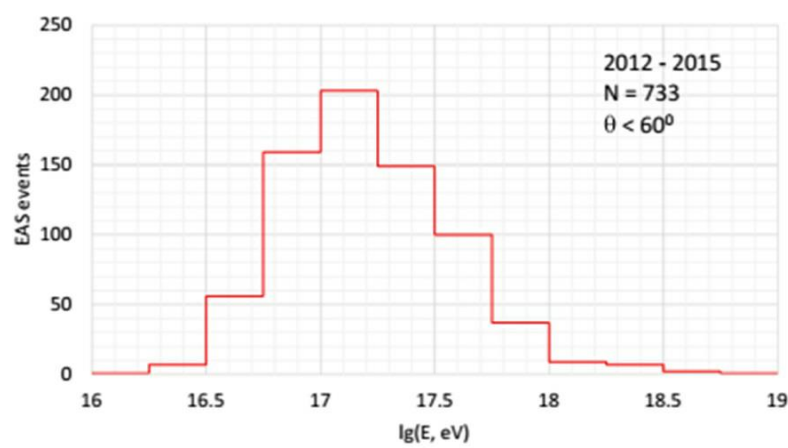

Figure 4: Energy distribution for EAS events detected by coincidence scintillation detectors of Yakutsk array and wide field of view Cherenkov telescope

photon density distribution functions are used to locate the shower core, the FWHM(R) function can be used additionally (or instead) to refine the core coordinates in the array plane. As an illustration, the signal width is shown as a function of energy in Fig. 6. A systematic variation of the width is visible, although the tendencies are contradictory in the shower core distance intervals. More data is needed, especially at distances to the shower core less than one hundred meters, to reveal the reliable energy dependence. Application of the FWHM(R) function is possible in the shower core location procedure in the array plane at least additionally to the Cherenkov photon density distribution.

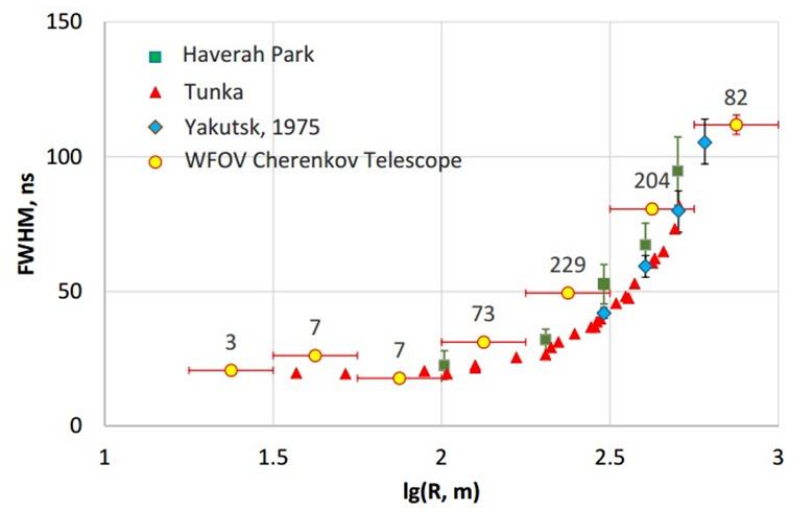

Figure 5: Full width at half-maximum of the Cherenkov signal as a function of the shower core distance, R, measured by EAS arrays

\section{Method to Estimate the Distance to the Shower Maximum via Cherenkov Light Measurements}

Common methods, which rely on Cherenkov light detectors to find the height in the atmosphere, $h_{\max }$, where the number of EAS particles reaches a maximum, are based on measurements of the lateral distribution of the photon density and pulse duration[16, 17, 18]. We propose another approach to evaluate $h_{\max }$ resulting from our measurements of the Cherenkov light induced by EAS. Knowing the time delay of the Cherenkov signal maximum in the detector relative to the 


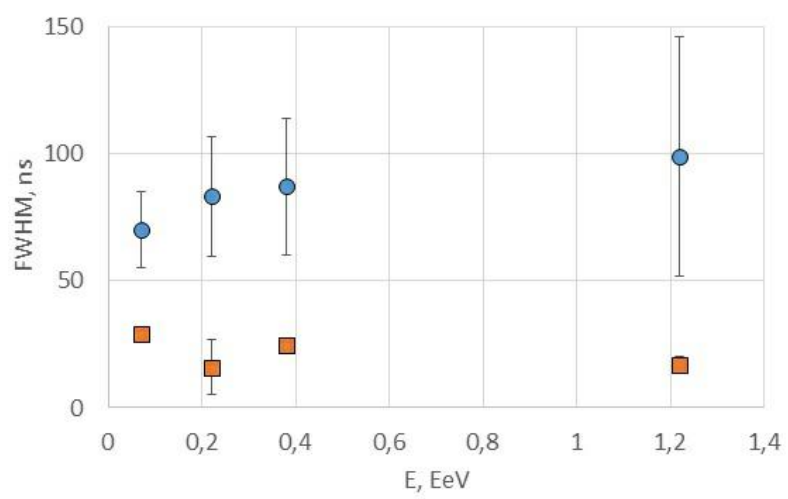

Figure 6: Energy dependence of Full Width at Half-Maximum of the Cherenkov signal

shower axis crossing the array plane at distance, $\mathrm{R}$, one is able to calculate the distance, $\mathrm{h}_{\theta}$, to the height in atmosphere, $\mathrm{h}_{\max }$, where the emission of Cherenkov photons reaches a maximum. Other shower parameters needed are the coordinates of the shower axis and the EAS arrival angles. In our case, these parameters can be provided by the set of synchronized surface detectors of the Yakutsk array. The time difference, $\Delta t$, is determined by triangles consisting of $\mathrm{R}, \mathrm{h}_{\max }, \mathrm{h}_{\theta}$. More information about this method can be found in the article [19].

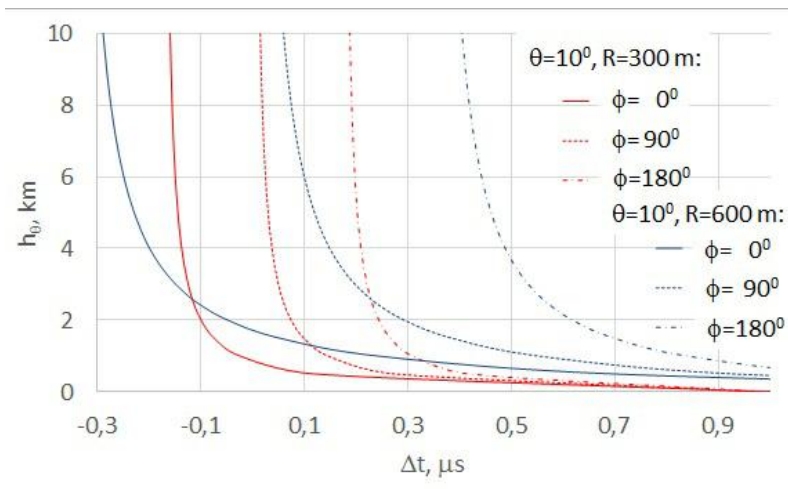

Figure 7: The height of Cherenkov light emission maximum as a function of time difference, $\Delta \mathrm{t}$, between Cherenkov photons arriving at the detector and to the shower core in the array plane. The azimuth angle, $\phi$, is between the detector and the shower axis projected on the array plane.

$$
c \Delta t=\sqrt{h_{\theta}^{2}+R^{2}-2 R h_{\theta} \sin \theta \cos \phi}-h_{\theta},
$$

where $\phi, \theta$ are shower arrival angles; $\mathrm{c}=0.3 \mathrm{~m} / \mathrm{ns}$. A solution is given by

$$
h_{\theta}=\frac{0.5\left(R^{2}-(c \Delta t)^{2}\right)}{c \Delta t+R \sin \theta \cos \phi}
$$

The solution of the equation is shown in Fig. 7. Rather large values of the derivative $\mathrm{dh}_{\theta} / \mathrm{d} \Delta \mathrm{t}$ near the time difference threshold restrict the maximum possible locations within narrow limits due to moderate timing accuracy. On the other hand, relatively small heights can be resolved with reasonable timing resolution. We illustrate in Fig. 8 the maximum height location accuracy 
achievable with the different shower core and time measurement errors. The present accuracy for the Yakutsk array detectors is not sufficient to measure the distance to the shower maximum with reliable results. But the Yakutsk array modernization program now in progress includes the best part of the improvements needed. Specifically, a target synchronization accuracy of detectors will equal $5 \mathrm{~ns}$ due to Gigabit Ethernet via optical fibers. New scintillation counters and Cherenkov light detectors will provide the core location accuracy of showers within $15-20 \mathrm{~m}$. Accordingly, the modernized array will be able to measure the Cherenkov $X_{\max }$ to an accuracy of $3-4 \%$, if the time resolution of the Cherenkov light detectors will be better than $5 \mathrm{~ns}$.

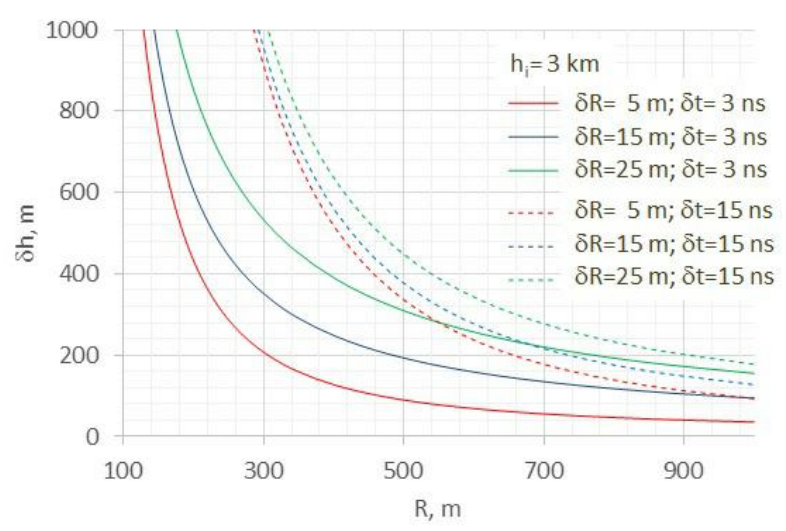

Figure 8: Estimation of the $h_{\max }$ reconstruction accuracy as a function of the shower core distance. Different EAS core location and timing errors are given.

\section{Conclusions}

A new approach is explored in estimating the distance to the shower maximum based on the time difference between Cherenkov photons arriving at the detector and the shower core in the array plane. Fast optical detectors and a nanosecond-order synchronization system of the array detectors are essential in future implementations of the method. A Cherenkov telescope prototype obtained experimental data for the period from 19.10.2012 to 29.03.2017, on 220 clear moonless nights with about 1670 hours of observation, during the observation of the master load 30,000 times, of which the Cherenkov telescope gave a signal to the coincidence of about 1450 times. Measuring the width at half-maximum as function of distance from the shower core, (R), will help determine the shower core in the array plane. Further modernization of the array will enable to calculate the height of the shower maximum by the time delay.

\section{Acknowledgments}

The reported study was funded by RFBR according to the research project 16-29-13019. Grant NOFMU "academic mobility" and Head of Sakha Republic Grant for young scientist 17/16.

\section{References}

[1] J.V. Jelley, Cherenkov Radiation and its Applications (Pergamon Press, 1958) 
[2] A.S. Lidvansky, Radiation Physics and Chemistry 7589 (2006)

[3] A.A. Watson, astro-ph/1101.4535.

[4] V.I. Zatsepin, J. Exp. Theor. Phys. 47689 (1964)

[5] Yu.A. Fomin and G.B. Khristiansen, Yad. Fiz. 14642 (1971)

[6] N.N. Kalmykov et al., J. Exp. Theor. Phys. Lett. 2166 (1975)

[7] R.T. Hammond et al., Nuovo Cim. C1 315 (1978)

[8] M.N. Dyakonov et al., Nucl. Instrum. Meth. A 248224 (1986)

[9] V.M. Grigorjev et al., J. Exp. Theor. Phys. Lett.30 727 (1979)

[10] S.F. Berezhnev et al., Nucl. Instrum. Meth. A 69298 (2012)

[11] E.G. Berezhko et al, Astropart. Phys. 3631 (2012)

[12] N.N. Kalmykov et al., J. Exp. Theor. Phys. Lett. 2166 (1975)

[13] R.T. Hammond et al., Nuovo Cim. C1 315 (1978)

[14] V.V. Prosin et al., Nucl. Phys. B - Proc. Suppl. 190247 (2009)

[15] J. Linsley, Proc. 19th ICRC 9434 (1985)

[16] M. Nagano and A.A. Watson, Rev. Mod. Phys. 72689 (2000) 689

[17] S.P. Knurenko and A.V. Sabourov, Astroph. Space Sci. Trans. 7251 (2011)

[18] E.G. Berezhko, S.P. Knurenko and L.T. Ksenofontov, Astropart. Phys. 3631 (2012)

[19] A.A. Ivanov for the Yakutsk array group, EPJ Web of Conf. 5304003 (2013)

[20] S.P. Knurenko, Z.E. Petrov, I.S. Petrov. Radio emission of air showers with extremely high energy measured by the Yakutsk Radio Array. Nuclear Instruments and Methods in Physics Research A. 866 (2017) 230-241. https://doi.org/10.1016/j.nima.2017.04.033 